\title{
Imaging: how to recognise idiopathic pulmonary fibrosis
}

\author{
Anand Devaraj \\ Affiliations: Dept of Radiology, St George's Hospital, London, UK. \\ Correspondence: Anand Devaraj, Dept of Radiology, St George's Hospital, Blackshaw Road, London, SW17 OQT, UK. \\ E-mail: a.devarajanhs.net
}

ABSTRACT It is well known that high-resolution computed tomography (HRCT) is an essential component of the diagnostic pathway in idiopathic pulmonary fibrosis (IPF). Honeycombing, a common feature of IPF seen on HRCT, is crucial for an accurate diagnosis. Unfortunately, identification of honeycombing is not always straightforward, and there is some disagreement regarding its imaging features. It can be difficult to distinguish honeycombing from traction bronchiectasis and emphysema, although several imaging characteristics can be helpful. Recently, there has been an interest in expanding the use of HRCT beyond diagnosis for disease monitoring and prognostication, and several studies have provided valuable contributions in this regard. Traction bronchiectasis and the extent of fibrosis, for example, have been reported to be powerful prognostic predictors for mortality. Finally, considering the difficulties in diagnosis of "possible usual interstitial pneumonia", clinicians should always be aware that clinical factors must be considered together with HRCT in order to reach an accurate diagnosis and provide appropriate treatment.

0

@ERSpublications

In addition to clinical factors, HRCT features are increasingly useful for both diagnosis and prognosis of IPF http://ow.ly/vAJJ8

\section{Introduction}

Idiopathic pulmonary fibrosis (IPF) has been defined by international guidelines as a specific form of chronic, progressive, fibrosing interstitial pneumonia of unknown cause, occurring primarily in older adults, limited to the lungs and associated with the histopathological and/or radiological pattern of usual interstitial pneumonia (UIP) [1]. Diagnosis of IPF poses several challenges for clinicians, and the accuracy of diagnosis increases with multidisciplinary team discussion between pulmonologists, radiologists and pathologists.

As is well-known, high-resolution computed tomography (HRCT) is an essential component of the diagnostic pathway in IPF. However, due to the heterogeneity of presentations of IPF, a radiological diagnosis of IPF using HRCT is not always straightforward, and the disease may have both typical and atypical appearances. In this regard, honeycombing, a common feature of IPF seen on HRCT, is crucial for an accurate diagnosis. Unfortunately, identification of honeycombing is often subjective, and there is some disagreement regarding its imaging features. More recently, there has been increasing interest in the use of HRCT beyond diagnosis in both disease monitoring and prognostication. This review will focus on the expanding role of computed tomography in these latter aspects, as well as the difficulties involved in the identification of honeycombing.

Received: Feb 282014 | Accepted after revision: April 022014

Conflict of interest: Disclosures can be found alongside the online version of this article at err.ersjournals.com

Provenance: Publication of this peer-reviewed article was sponsored by InterMune International AG, Muttenz, Switzerland (article sponsor, European Respiratory Review issue 132).

Copyright OERS 2014. ERR articles are open access and distributed under the terms of the Creative Commons Attribution Non-Commercial Licence 4.0. 


\section{Diagnostic criteria}

According to international guidelines [1], the diagnosis of IPF requires the following: 1) exclusion of other known causes of interstitial lung disease, e.g. domestic and occupational environmental exposures, connective tissue disease and drug toxicity; 2) the presence of a definite UIP pattern on HRCT in patients not subjected to surgical lung biopsy; and 3) specific combinations of HRCT and surgical lung biopsy patterns in patients subjected to surgical lung biopsy.

A definite UIP pattern on HRCT is present if the following four radiological criteria are met: 1) subpleural basal predominance; 2) reticular abnormality; 3) honeycombing with or without traction bronchiectasis; and 4) absence of features listed as inconsistent with UIP pattern.

For patients who do not meet the criteria for a definite UIP pattern, there are two other classifications: a possible UIP pattern or an inconsistent with UIP pattern (table 1).

\section{Typical appearance of UIP on HRCT}

The distribution of honeycombing in UIP on HRCT is typically basal and peripheral (fig. 1). Ground-glass opacities are sparse or absent. Another HRCT characteristic that can be helpful in making a diagnosis of UIP is the so-called "propeller blade" distribution. This variant gets its name from the fact that, when seen on sequential axial images from caudal to cranial, the honeycombing or reticulation changes from being predominantly posterior to anterior [2].

\section{The challenges associated with identification of honeycombing}

Formal definitions of honeycombing that exist in the literature can help with standardising the identification of honeycombing. The Fleischner Society glossary provides both radiological and pathological definitions [3]. Radiologically, honeycombing is characterised by "clustered cystic air spaces, cysts of comparable diameters, and cyst diameters typically $<10 \mathrm{~mm}$ surrounded by well-defined walls" [3]. The pathology is defined as "destroyed and fibrotic lung tissue containing numerous cystic airspaces with thick fibrous walls, representing the late stage of various lung diseases, with complete loss of acinar architecture" [3].

Multi-layered cystic air spaces are not specifically part of the radiological definition, although some clinicians feel this is a requirement to more confidently describe the presence of honeycombing [4]. This highlights the significant degree of variation in actual working definitions used by radiologists, as demonstrated in a recent study by WATADANI et al. [5]. In this study, 43 observers rated 80 HRCT images using a five-point scale for honeycombing; only moderate agreement was observed. In $29 \%$ of cases, there was disagreement on identification of honeycombing, which was largely caused by conditions that mimic honeycombing, including mainly superimposed pulmonary emphysema.

One of the other problematic areas identified by the study was in differentiating honeycombing from traction bronchiectasis. It should be noted that scrolling through contiguous thin-section axial images can help identify a dilated airway, and thus traction bronchiectasis; coronal reformats may also be of use in this regard. An example in which identification of honeycombing was rendered difficult by the presence of emphysema is shown in figure 2. In general, honeycombing can be regarded as being more thick-walled,

\section{TABLE 1 High-resolution computed tomography criteria for usual interstitial pneumonia (UIP) pattern}

\section{UIP pattern (all four features)}

Subpleural, basal predominance Reticular abnormality

Honeycombing with or without traction bronchiectasis

Absence of features listed as inconsistent with UIP pattern

Possible UIP pattern (all three features)

Inconsistent with UIP pattern lany of the features]

Subpleural, basal predominance Reticular abnormality

Absence of features listed as inconsistent with UIP pattern
Upper or mid-lung predominance

Peribronchovascular predominance

Extensive ground-glass abnormality lextent $>$ reticular abnormalityl

Profuse micronodules (bilateral, predominantly upper lobes)

Discrete cysts (multiple, bilateral, away from areas of honeycombing)

Diffuse mosaic attenuation/air-trapping (bilateral and in $\geqslant 3$ lobes)

Consolidation in bronchopulmonary segment(s)/lobels)

Reproduced from [1] with permission from the publisher. 
FIGURE 1 High-resolution computed tomography scan showing typical appearance of usual interstitial pneumonia.

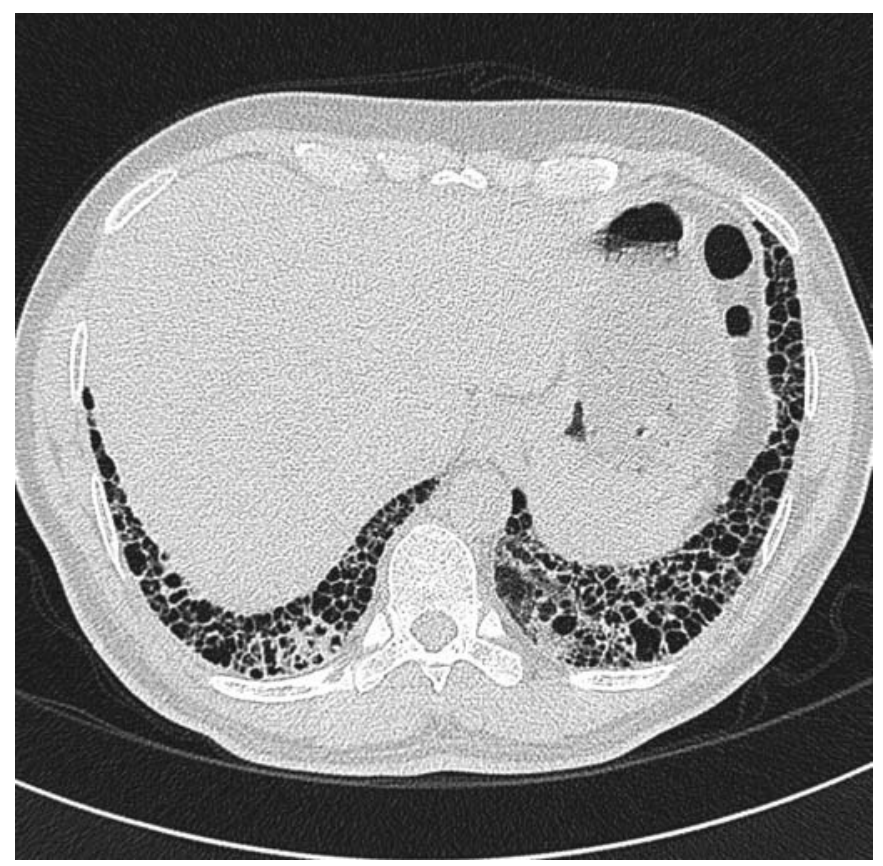

subpleural and parallel to the chest wall. Emphysema is typically characterised by thinner walls and cystic airspaces that have a propensity to be located further away from the chest wall [6].

\section{Differential diagnosis of atypical IPF on HRCT}

The differential diagnosis of IPF by HRCT is further complicated by the fact that biopsy-proven UIP may mimic other interstitial disease including, in order of frequency, nonspecific interstitial pneumonia (NSIP), chronic hypersensitivity pneumonitis and sarcoidosis [7]. The typical HRCT features of NSIP include peripheral basal or peribronchovascular ground-glass opacities, which may be associated with traction bronchiectasis. Honeycombing is sparse or absent. Other features that are inconsistent with a diagnosis of UIP include air trapping, segmental consolidation, upper lobe predominance, peribronchovascular reticulation and micronodules.

Based on the consensus statement from the American Thoracic Society/European Respiratory Society/ Japanese Respiratory Society/Latin American Thoracic Association [1], the term "possible UIP" has been introduced. This should be considered in the presence of basal subpleural reticular predominant abnormalities, where honeycombing is absent and where there is an absence of atypical features. This term may sometimes be favoured in patients in whom biopsy is required to confirm the diagnosis. However, an alternative approach for radiologists to consider, in cases where HRCT features are not typical of UIP, is to provide a differential diagnosis of the appearance together with an expression of likelihood. This approach allows clinicians to integrate clinical factors, including age and comorbidities, before deciding upon the need for invasive biopsy.

Considering fibrotic idiopathic interstitial pneumonias (IIPs) with little honeycombing, the results from two recent publications have provided guidance on differentiating UIP from NSIP without biopsy [8, 9]. In particular, UIP has been shown to be associated with increasing age, increasing reticulation and decreasing ground-glass opacities. Thus, in some scenarios, it could be suggested that a combination of age and extent of reticulation may be sufficient to change the diagnosis in the clinicians mind from "possible UIP" to "probable UIP".

\section{Using HRCT to prognosticate IPF and monitor disease progression}

There is increasing interest in using patterns found on HRCT as prognostic determinants. A study by SUMIKAWA et al. [10] investigated correlations between HRCT findings and mortality in 98 patients with a histological diagnosis of UIP and a clinical diagnosis of IPF. Patients were grouped as follows based on the results of HRCT scans: 1) definite UIP; 2) consistent with UIP; and 3) suggestive of an alternative diagnosis. While there was no difference in survival between groups, prognosis was found to be significantly influenced by the extent of traction bronchiectasis and fibrosis. 


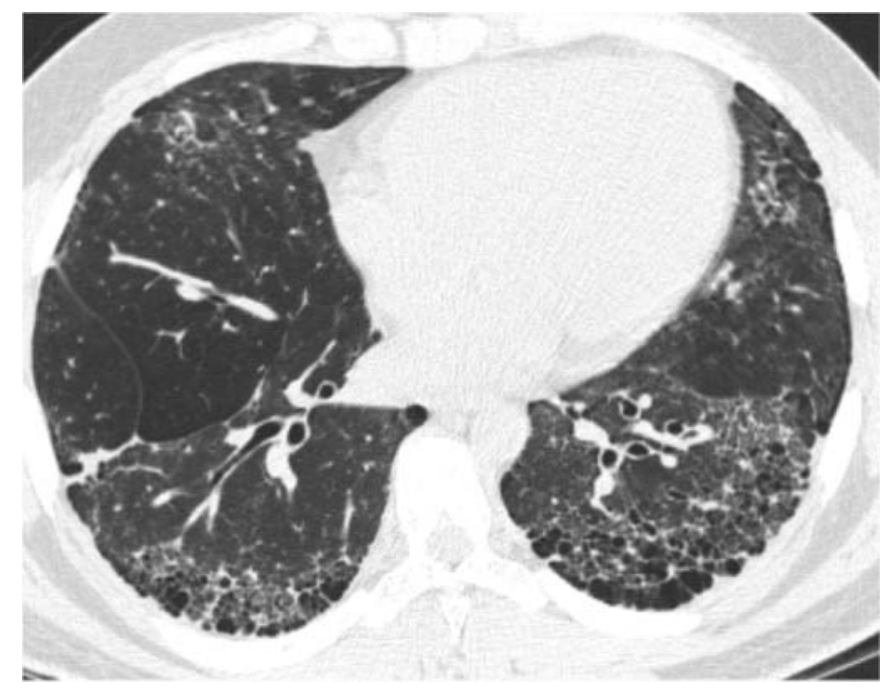

FIGURE 2 Computed tomography scan from a patient with emphysema, thus identification of honeycombing was rendered difficult.

A more recent study by WALSH et al. [11] confirmed that HRCT patterns can be useful prognostic determinants of mortality. By examining the HRCT scans from 168 patients with connective tissue diseaserelated fibrotic lung disease, the authors reported that the severity of traction bronchiectasis, extent of honeycombing and reduction in diffusing capacity of the lung for carbon monoxide were all strongly and independently associated with increased mortality in connective tissue disease-related fibrotic lung disease. While providing important prognostic information, it can be speculated that traction bronchiectasis is a good predictor of mortality as it may be associated with a greater degree of fibrosis at the microscopic level.

LEE et al. [9] used HRCT to monitor disease progression in 154 patients (UIP: $n=101$; NSIP: $n=53$ ) with fibrotic IIPs with little honeycombing and a histological diagnosis of a fibrotic IIP $(<5 \%$ honeycombing on HRCT). The patients were followed clinically for at least 2 years. On serial scans, honeycombing (UIP: $+5 \%$; fibrotic NSIP: $+3 \%$ ) and reticulation (UIP: $+3 \%$; fibrotic NSIP: $+8 \%$ ) significantly progressed in extent, while ground-glass opacities (UIP: -2\%; fibrotic NSIP: $-10 \%$ ) decreased in extent. The overall extent of lesions increased in UIP (+6\%) and decreased in NSIP (-4\%). Interestingly, among patients with UIP, $14 \%$ had improved pulmonary function test results, $47 \%$ had stable results and $38 \%$ had worse results. Thus, stable disease was the most common scenario. Moreover, changes in the extent of fibrosis correlated only modestly with changes in forced vital capacity $(r=0.45)$, and poorly with changes in diffusing capacity of the lung for carbon monoxide $(r=0.24)$. The overall extent of lung parenchymal abnormalities and reticulation on baseline HRCT, changes in overall extent and the development of honeycombing on serial HRCT were associated with shorter patient survival in univariate analysis. However, in multivariate analysis only the overall extent of lung fibrosis on the baseline HRCT helped predict survival in fibrotic IIPs with little honeycombing.

The ability of HRCT to identify changes in disease extent over time has led other investigators to evaluate the relationship between disease progression on serial HRCT and overall prognosis. HWANG et al. [12] evaluated the prognostic implications of HRCT and physiological variables at baseline and after sequential evaluation in 72 patients with fibrosing interstitial pneumonia (idiopathic disease: $n=42$, collagen vascular disease: $n=30$ ). On follow-up HRCT, the extent of mixed ground-glass and reticular opacities, pure reticular opacity, honeycombing and overall extent of disease were all significantly increased in the idiopathic group, but remained unchanged in the collagen vascular disease group. Importantly, the extent of honeycombing at baseline and its progression on follow-up HRCT were both found to be important predictors of mortality in patients with fibrosing interstitial pneumonia.

Subjects who demonstrated progression of fibrosis extent on HRCT over a period of 6 months and a decline in forced vital capacity were identified in a study by ODA et al. [13] as a particular sub-group of IPF patients with the poorest outcomes. In fact this was shown to be a superior predictor of outcome compared to baseline HRCT fibrosis scores [13].

\section{Conclusion}

The typical features of UIP include the presence of honeycombing, although this can sometimes be difficult for clinicians to confidently identify and agree upon. Indeed, some of the most problematic areas are differentiating honeycombing from traction bronchiectasis and emphysema, although there are criteria that can help radiologists in correctly identifying UIP. Differentiating UIP from NSIP can also be challenging on 
HRCT, but this may be facilitated by recognising features that are inconsistent with a diagnosis of the former such as predominance of ground-glass opacities and a peribronchovascular distribution. When considering a diagnosis, the term "possible UIP" may be preferred in some cases as it highlights patients in whom a biopsy should be considered for a more definitive diagnosis. Recent studies have suggested that HRCT may be of prognostic value for IPF patients. This has been demonstrated both at baseline computed tomography, where traction bronchiectasis is a powerful prognostic predictor, and on follow-up computed tomography where progression of fibrosis and honeycombing is associated with poorer outcomes.

\section{Acknowledgements}

This article is based on the proceedings of the 2013 Advancing IPF Research (AIR) meeting (Nice, France), which was sponsored by InterMune International AG (Muttenz, Switzerland). Medical writing support was provided by Michael Smith (IntraMed International, Milan, Italy), which was funded by InterMune International AG.

\section{References}

1 Raghu G, Collard HR, Egan JJ, et al. An official ATS/ERS/JRS/ALAT statement: idiopathic pulmonary fibrosis: evidence-based guidelines for diagnosis and management. Am J Respir Crit Care Med 2011; 183: 788-824.

2 Hunninghake GW, Lynch DA, Galvin JR, et al. Radiologic findings are strongly associated with a pathologic diagnosis of usual interstitial pneumonia. Chest 2003; 124: 1215-1223.

3 Hansell DM, Bankier AA, MacMahon H, et al. Fleischner Society: glossary of terms for thoracic imaging. Radiology 2008; 246: 697-722.

4 Johkoh T, Sakai F, Noma S, et al. Honeycombing on CT: its definitions, pathologic correlation, and future direction of its diagnosis. Eur J Radiol 2014; 83: 27-31.

5 Watadani T, Sakai F, Johkoh T, et al. Interobserver variability in the $\mathrm{CT}$ assessment of honeycombing in the lungs. Radiology 2013; 266: 936-944.

Arakawa H, Honma K. Honeycomb lung: history and current concepts. AJR Am J Roentgenol 2011; 196: 773-782. Sverzellati N, Wells AU, Tomassetti S, et al. Biopsy-proved idiopathic pulmonary fibrosis: spectrum of nondiagnostic thin-section CT diagnoses. Radiology 2010; 254: 957-964.

8 Fell CD, Martinez FJ, Liu LX, et al. Clinical predictors of a diagnosis of idiopathic pulmonary fibrosis. Am J Respir Crit Care Med 2010; 181: 832-837.

9 Lee HY, Lee KS, Jeong YJ, et al. High-resolution CT findings in fibrotic idiopathic interstitial pneumonias with little honeycombing: serial changes and prognostic implications. AJR Am J Roentgenol 2012; 199: 982-989.

10 Sumikawa H, Johkoh T, Colby TV, et al. Computed tomography findings in pathological usual interstitial pneumonia: relationship to survival. Am J Respir Crit Care Med 2008; 177: 433-439.

11 Walsh SL, Sverzellati N, Devaraj A, et al. Connective tissue disease related fibrotic lung disease: high resolution computed tomographic and pulmonary function indices as prognostic determinants. Thorax 2014; 69: 216-222.

12 Hwang JH, Misumi S, Curran-Everett D, et al. Longitudinal follow-up of fibrosing interstitial pneumonia: relationship between physiologic testing, computed tomography changes, and survival rate. J Thorac Imaging 2011; 26: 209-217.

13 Oda K, Ishimoto H, Yatera K, et al. High-resolution CT scoring system-based grading scale predicts the clinical outcomes in patients with idiopathic pulmonary fibrosis. Respir Res 2014; 15: 10. 